\title{
$\underline{\mathbf{P}-133}$
}

\section{Rapid Identification of Oligostilbenes in Dipterocarpaceae Crude Extract}

\author{
Ratni Suriyani Jalal ${ }^{1}$, Norizan Ahmat $^{2}$, Aizam Ekhmal Esahak Ayub ${ }^{1}$, Mohd Shafarin Shafie ${ }^{1}$,
} Qamarusy Syazwan Kamarul Bahrin ${ }^{1}$ and Nurhuda Manshoor ${ }^{1, *}$

${ }^{1}$ Atta-ur-Rahman Institute for Natural Products Discovery (RiND), Faculty of Pharmacy, Universiti Teknologi MARA, 42300, Puncak Alam, Selangor, Malaysia; ${ }^{2}$ Atta-ur-Rahman Institute for Natural Products Discovery (RiND), Faculty of Applied Sciences, Universiti Teknologi MARA, 40450, Shah Alam, Selangor, Malaysia; E-mail: nurhuda15@puncakalam.uitm.edu.my

A rapid and systematic tandem LC-MS protocol is utilized to identify oligostilbenes in three Dryobalanops species (D. rappa, D. lanceolata and D. aromatica; family Dipterocarpaceae). Analyses were performed by a newly developed dereplication system on a liquid chromatography-ion trap-mass spectrometry (LC-IT-MS) with electrospray ionization (ESI) interface. The combination of effective separation by an HPLC and multiple fragmentation steps for specific masses in MS enable fast and sensitive analysis of various degrees of oligostilbenes condensation.

Keywords: LC-MS ${ }^{\mathrm{n}}$, oligostilbenes, ESI, ion trap, Dipterocarpaceae. 